\title{
CAROTENOIDES EM ABÓBORAS
}

CAROLINA MÉDICI VERONEZI*

NEUZA JORGE**

\begin{abstract}
A presente revisão de literatura evidencia a importância dos carotenoides, como compostos bioativos e seu potencial para aplicação em alimentos funcionais. Foram detalhadas a origem, as características químicas e as fontes de carotenoides. Também foram estudadas as metodologias mais utilizadas na sua quantificação e qualificação, incluindo a espectrofotometria, a espectrometria de massa, a cromatografia a líquido de alta eficiência, a cromatografia a gás e a colorimetria. Recomenda-se a ingestão de abóboras por apresentarem quantidades relevantes de importantes carotenoides, como $\alpha$-caroteno, $\beta$-caroteno e luteína.
\end{abstract}

PALAVRAS-CHAVES: CAROTENOIDES; ABÓBORAS; ANTIOXIDANTES; PRÓ-VITAMINA A.

* Mestranda em Engenharia e Ciência de Alimentos, Instituto de Biociências, Letras e Ciências Exatas (IBILCE), Universidade Estadual Paulista (UNESP), São José do Rio Preto, SP (e-mail: cveronezi@ hotmail.com).

** Professora Adjunta, Departamento de Engenharia e Tecnologia de Alimentos, IBILCE/UNESP, São José do Rio Preto, SP (e-mail: njorge@ibilce.unesp.br). 


\section{INTRODUÇÃo}

Os carotenoides assumem grande destaque na alimentação humana como fonte de vitamina $A$ e pela sua ação antioxidante que está relacionada com a diminuição de riscos de doenças degenerativas (como as cardiovasculares, câncer e obesidade, dentre outras). Estão presentes em tecidos animais, fungos e bactérias. Porém, as principais fontes de carotenoides e as mais importantes para a saúde humana são as frutas e demais vegetais in natura, dentre os quais as abóboras (família das Cucurbitaceaes). A área de cultivo da abóbora no Brasil ultrapassa 20 mil hectares, embora a maior parte da produção seja destinada à exportação. As abóboras são muito apreciadas e consumidas sob as formas de doces ou em diversos pratos salgados.

Os carotenoides podem ser quantificados e qualificados em alimentos por várias técnicas, como espectrofotometria, espectrometria de massa, cromatografia a líquido de alta eficiência, cromatografia a gás e colorimetria.

Neste trabalho foram abordados os carotenoides mediante revisão sobre sua origem, características, funções e metodologias de quantificação e qualificação, enfocando aqueles encontrados em abóboras.

\section{ORIGEM DOS CAROTENOIDES}

Os estudos sobre os carotenoides tiveram início no século XIX, sendo os primeiros isolados de cenouras em 1831. Existem mais de 600 carotenoides identificados, sendo que aproximadamente $10 \%$ são encontrados na dieta humana e $20 \%$ no plasma e tecidos de mamíferos (HUGHES, 1994; ÖTLES e ÇAGINDI, 2007; ROCK, 1997). Como resultado da absorção seletiva do trato gastrointestinal, apenas 14 carotenoides são biodisponíveis (COOPER, ELDRIDGE e PETERS, 1999).

Os carotenoides são considerados compostos bioativos, além de serem lipossolúveis, poliinsaturados, sintetizados unicamente por vegetais, formando um dos mais importantes grupos de pigmentos naturais encontrados na natureza (OLIVER e PALOU, 2000; ÖTLES e ÇAGINDI, 2007; SANDERS, 1994).

\section{ESTRUTURA QUÍMICA}

Estruturalmente, os carotenoides são poliisoprenoides compostos por quarenta átomos de carbonos simétricos e lineares, formados pela união cauda-cabeça de oito unidades isoprenoides, exceto na posição central (ÖTLES e ÇAGINDI, 2007). Apresentam amplo sistema de duplas ligações conjugadas e por isso podem ser oxidados tanto química quanto biologicamente (RODRIGUEZAMAYA, KIMURA e AMAYA-FARFAN, 2008).

A nomenclatura sistemática dos carotenoides baseia-se no núcleo central denominado caroteno, sendo as especificações para as extremidades designadas pelas letras gregas $\beta$ (beta), $\dot{\varepsilon}$ (épsilon), $\psi$ (psi), $\xi$ (phi) e k (kappa). Tais letras assumem os seguintes significados: $\beta$ e $\dot{\varepsilon}$, ciclohexano; k, ciclopenteno; $\psi$, acíclico e $\xi$, cíclico (PIMENTEL, FRANCKI e GOLLÜCKE, 2005). O esqueleto básico dessas moléculas pode sofrer modificações, devido à ciclização, hidrogenação, desidrogenação, introdução de grupos contendo oxigênio, rearranjos, encurtamento de cadeias ou combinações dessas modificações (DUTTA, CHAUDHURI e CHAKRABORTY, 2005; RODRIGUEZAMAYA, KIMURA e AMAYA-FARFAN, 2008). Como consequência ocorrem a perda de cor, da atividade biológica e a formação de compostos voláteis que provocam sabores indesejáveis, embora sejam moderadamente termoestáveis (RODRIGUEZ- AMAYA, 1999).

Quanto à estrutura química, os carotenoides são classificados em dois grupos distintos. Os chamados carotenos (hidrocarotenoides) apresentam somente átomos de carbono e hidrogênio em sua molécula e os chamados xantofilas (oxicarotenoides) contêm átomos de oxigênio em sua molécula, além de carbono e hidrogênio (OLIVER e PALOU, 2000; RODRIGUEZ-AMAYA, 1999; THANE e REDDY, 1997). Os grupos substituintes oxigenados mais comuns são os de hidroxilas $(\mathrm{OH})$, ceto $(\mathrm{C}=\mathrm{O})$, epóxido, aldeído $(\mathrm{CHO})$, carboxi $\left(\mathrm{CO}_{2} \mathrm{H}\right)$, carbometoxi $\left(\mathrm{CO}_{2} \mathrm{Me}\right)$ e metoxi $(\mathrm{OMe})$ (RODRIGUEZ-AMAYA, KIMURA e AMAYA-FARFAN, 2008).

Quanto à funcionalidade, os carotenoides podem ser divididos em primários e secundários. Os primários são utilizados no processo fotossintético, como o $\beta$-caroteno e a luteína, enquanto os secundários não estão diretamente envolvidos na sobrevivência das plantas, como o a-caroteno e 
o licopeno (MATTEA, MARTÍN e COCERO, 2009). Podem também ser classificados em acíclicos, monocíclicos ou bicíclicos. Essa ciclização ocorre em um ou ambos extremos da molécula, formando um ou dois anéis $\beta$ de seis átomos ou anéis $\varepsilon$ (RODRIGUEZ-AMAYA, KIMURA e AMAYA-FARFAN, 2008).

Os carotenoides são encontrados principalmente na forma trans, que é mais estável, porém a presença de alguns fatores físicos e/ou químicos (como luz solar direta, pró-oxidantes e calor) pode provocar modificação na molécula (SIQUEIRA et al., 2007).

Os principais carotenoides encontrados nos vegetais são: $\alpha$ e $\beta$-caroteno, luteína e licopeno (MEYERS, 1994). Suas estruturas estão demonstradas na Figura 1.<smiles>CC1=CCCC(C)(C)C1/C=C/C(C)=C/C=C/C(C)=C/C=C/C=C(C)/C=C/C=C(C)/C=C/C1=C(C)CCCC1(C)C</smiles><smiles>CC1=C(/C=C/C(C)=C/C=C/C(C)=C/C=C/C=C(C)/C=C/C=C(C)/C=C/C2=C(C)CCCC2(C)C)C(C)(C)CCC1</smiles>

FIGURA 1 - ALGUNS CAROTENOIDES

Fonte: adaptado de AMBRÓSIO, CAMPOS e FARO, 2006.

\section{FONTES}

Os carotenoides são biossintetizados por plantas. Os alimentos de origem vegetal contêm, além dos carotenoides principais, pequenas quantidades de precursores e derivados, que tornam sua composição complexa e variável. Já os alimentos de origem animal não apresentam a mesma riqueza, pois são incapazes de biossintetizar carotenoides e, por isso, dependem da sua ingestão (RODRIGUEZ-AMAYA, KIMURA e AMAYA-FARFAN, 2008).

A composição dos carotenoides de frutas e hortaliças é afetada por fatores como a variedade, desigualdade na distribuição em dado alimento ou parte do vegetal que é consumido, grau de maturação, tipo de solo, área geográfica da produção, condições de colheita, processamento e armazenamento (BERNHARDT e SCHLICH, 2006; SHILLS et al., 2003).

Os vegetais folhosos ou não folhosos apresentam perfil de carotenoides qualitativo definido, sendo a luteína, o $\beta$-caroteno, a violaxantina e a neoxantina os principais. Já as frutas e os vegetais frutos têm composição em carotenoides bem mais complexa e diversificada, destacando-se o $\alpha$-caroteno, o $\beta$-caroteno e a luteína (RODRIGUEZ-AMAYA, KIMURA e AMAYA-FARFAN, 2008). A Tabela 1 mostra algumas fontes de carotenoides.

\section{CAROTENOIDES EM ABÓBORAS}

Dentre a grande variedade de frutos produzidos no Brasil encontram-se os pertencentes à ordem Cucurbitales, gênero Cucurbita e família Cucurbitaceaes que compreende mais de 800 espécies distribuídas em todo o mundo. Essa família inclui os pepinos, os melões, as melancias, além de todas as hortaliças popularmente denominadas de abóboras (ZITTER, HOPKINS e THOMAS, 1996). 
TABELA 1 - FONTES DE CAROTENOIDES

\begin{tabular}{lccc}
\hline \multirow{2}{*}{ Descrição } & & Carotenoides $(\boldsymbol{\mu g} \mathbf{g})$ & \\
\cline { 2 - 4 } & $\alpha$-caroteno & $\beta$-caroteno & luteína \\
\cline { 2 - 4 } & & 112 & 119 \\
\hline Vegetais Folhosos ou não & 4,7 & 66 & 104 \\
\hline Caruru & 7,1 & 81 & 4,9 \\
Taioba & 34 & & 1,6 \\
Cenoura Nantes & & 5,4 & 0,4 \\
\hline Frutas & 0,5 & 1,6 & 0,7 \\
\hline Acerola & 0,9 & 7,0 & 18 \\
Cajá & 0,6 & & 10 \\
Marolo & & 57 & 10 \\
\hline Vegetais Frutos & 24 & 53 & 16 \\
\hline Abóbora Goianinha & 25 & 0,1 & \\
Abóbora Menina & & & \\
Abóbora Moranga Exposição & & & \\
\hline
\end{tabular}

Fonte: RODRIGUEZ-AMAYA, KIMURA e AMAYA-FARFAN (2008).

A Cucurbita moschata (abóbora rasteira) e a Cucurbita máxima (morangas) são as espécies de maior valor nutricional e agroeconômico, além da Cucurbita pepo (FILGUEIRA, 2000), que diferem quanto ao formato, tamanho, cor da casca, cor da polpa, firmeza, teor de amido, teor de matéria seca e sabor (LUENGO e CALBO, 2001).

Sob o ponto de vista nutricional, a importância da presença de abóboras na dieta reside no fato de serem consideradas fontes de carotenoides, compostos responsáveis pela sua atrativa coloração e que desempenham importante papel na saúde humana (ZITTER, HOPKINS e THOMAS, 1996).

Arima e Rodriguez-Amaya (1990), após avaliarem a composição em carotenoides de abóboras provenientes do Nordeste brasileiro, identificaram que a Cucurbita moschata, variedade baianinha, apresenta 19 tipos de carotenoides, sendo 74\% (317,8 $\mu \mathrm{g} / \mathrm{g}) \beta$-caroteno. Já a Cucurbita máxima, variedade Jerimum caboclo, apresenta 11 tipos de carotenoides, sendo $60 \%$ luteína e $27 \%$ $\beta$-caroteno.

Murkovic, Mülleder e Neunteufl (2002), avaliando espécies de abóboras austríacas, afirmaram que a Cucurbita moschata tem variação em carotenoide de 0,98-5,9 mg/100 g de a-caroteno, 3,1-7mg/100 g de $\beta$-caroteno e 0,08-1,1 mg/100 $\mathrm{g}$ de luteína e zeaxantina. Já na Cucurbita maxima foi encontrado de 0-7,5 mg/100 $\mathrm{g}$ de $\alpha$-caroteno, 1,4-7,4 mg/100 $\mathrm{g}$ de $\beta$-caroteno e 0,8-17 mg/100 g de luteína e zeaxantina. A Cucurbita pepo apresenta 0,03-0,15 mg/100 g de a-caroteno, 0,06-2,3 mg/100 g de $\beta$-caroteno e 0-1,8 de luteína e zeaxantina.

Kandlakunta, Rajendran e Thingnganing (2008) avaliaram o conteúdo de carotenoides totais e $\beta$-caroteno de várias fontes de cereais, vegetais e condimentos. Verificaram a presença de $2120 \mu \mathrm{g}$ de carotenoides totais e $1180 \mu \mathrm{g}$ de $\beta$-caroteno em $100 \mathrm{~g}$ de abóboras amarelas (Cucurbita maxima). Aruna, Mamatha e Baskaran (2009), avaliando a porção de $100 \mathrm{~g}$ de abóboras Cucurbita máxima de variedade indiana, encontraram $10620 \mu \mathrm{g}$ de luteína e $278 \mu \mathrm{g}$ de zeaxantina. Ambos afirmaram que a abóbora pode ser considerada fonte de carotenoides.

Seo et al. (2005), avaliando a Cucurbita moschata, encontraram de $10-12 \%$ de $\alpha$-caroteno, $55-79 \%$ de $\beta$-caroteno e de $2-3 \%$ de luteína. Shi et al. (2010), após avaliarem a composição de carotenoides em abóboras (Cucurbita moschata), usando o método de extração por fluido supercrítico de $\mathrm{CO}_{2}$ e extração por solventes (etanol: hexano, 1:1), verificaram que os solventes têm maior poder de extração e encontraram $19,7 \%$ de $\alpha$-caroteno, $41,4 \%$ de $\beta$-caroteno e $35,3 \%$ de luteína.

A variação dos resultados decorre do processo de preparação da matéria-prima, das diferentes variedades e do estado de maturação do fruto (SHI et al., 2010).

Dentre os carotenoides presentes nas abóboras, os principais encontrados são o $\alpha$-caroteno (0-47 $\mu \mathrm{g} / \mathrm{g}$ de abóbora), o $\beta$-caroteno (1,4-235 $\mu \mathrm{g} / \mathrm{g}$ de abóbora) e a luteína (0-47 $\mu \mathrm{g} / \mathrm{g}$ de abóbora) (RODRIGUEZ-AMAYA; KIMURA; AMAYA-FARFAN, 2008). 


\section{1 a-CAROTENO}

Os $\alpha$-carotenos são importantes na dieta humana, pois atuam na formação de pigmentos visuais, no crescimento celular normal, no desenvolvimento e manutenção da estrutura epitelial e das mucosas que revestem os intestinos e as vias respiratórias, bem como no desenvolvimento de dentes e ossos. Só são biologicamente ativos quando transformados em vitamina A. Sua diferença em relação aos $\beta$-carotenos é a posição da dupla ligação do anel benzênico terminal (AMBRÓsıO, CAMPOS e FARO, 2006).

Os $\alpha$-carotenos são descritos como supressores da tumorogênese na pele, no pulmão, no fígado e no cólon, tendo atividade de supressão superior à promovida pelo $\beta$-caroteno. Agem promovendo a cessação do ciclo de multiplicação celular. Sua atuação pode ser identificada, tanto no estágio de iniciação quanto na promoção do câncer (NISHINO et al., 2002).

Estudos experimentais realizados em ratos induzidos ao câncer de pele mostraram que o grupo de animais que recebeu o suplemento de $\alpha$-caroteno apresentou, em média, apenas 0,3 tumores por rato-teste quando comparado ao grupo controle, 3,7 tumores por rato (NISHINO et al., 2002).

\section{$5.2 \beta$-CAROTENO}

Os $\beta$-carotenos são carotenoides bicíclicos, com potência vitamínicaAe bastante distribuídos na natureza. Aparecem na forma de dois isômeros geométricos, o trans e o cis, em proporções desiguais. São capazes de prevenir danos celulares, diminuir os níveis de espécies de oxigênio reativos no meio intracelular, reduzindo os riscos de lesão do material genético e promovendo ação antioxidante em células pulmonares expostas às nitrosaminas específicas do tabaco (BURRI, 1997; ÖTLES e ÇAGINDI, 2007).

Os $\beta$-carotenos não apresentam toxicidade detectável, mesmo se ingeridos em altas doses (RODRIGUEZ-AMAYA, 1999). Isso é possível pelo fato de não serem absorvidos pelo intestino e terem sua eficiência de absorção reduzida com o aumento da ingestão, além da taxa de conversão em vitamina A ser lenta sob o ponto de vista enzimático. No entanto, a ingestão excessiva pode causar hipercarotenose. A coloração amarela ou laranja da pele afeta as áreas em que a secreção gordurosa é mais intensa e nas superfícies queratinizadas. Estudos epidemiológicos também revelaram que a ingestão de $\beta$-caroteno está inversamente relacionada com a redução da incidência de alguns tipos de cânceres, como os de pulmão, pescoço e estômago, melhorando a taxa de sobrevida em pacientes com câncer de mama (SHILLS et al., 2003).

\subsection{LUTEÍNA}

A luteína é formada por uma molécula de a-caroteno e dois radicais hidroxilas, tendo a fórmula química $\mathrm{C}_{40} \mathrm{H}_{56} \mathrm{O}$. Apresenta coloração amarela e pode ser encontrada em muitas variedades de frutas, vegetais e óleos vegetais (ÖTLES e ÇAGINDI, 2007). É considerada potente antioxidante que previne danos causados pelos radicais livres nos tecidos. Esse carotenoide absorve a luz azul prejudicial aos olhos e com isso reduz em $40 \%$ a incidência de luz danosa à mácula, região responsável pela visão nítida de imagens (LADRUM e BONE, 2001; RODRIGUEZ-AMAYA, KIMURA e AMAYA-FARFAN, 2008; SILVA, 2004). Além disso, previne a aterosclerose, catarata, câncer de cólon e outras patologias (ÖTLES e ÇAGINDI, 2007; SILVA, 2004).

A ingestão de luteína recomendada é de $6 \mathrm{mg} / \mathrm{dia}$, sendo utilizada em produtos como sucos e tortas, alimentação de aves e na indústria de alimentos funcionais (SILVA, 2004).

Estudos demonstraram que ratos induzidos ao câncer de pele e suplementados com luteína tiveram incidência significativamente menor, quando comparados aos que não receberam o suplemento e evidenciaram ação anticarcinogênica (NISHINO et al., 2002). Outras pesquisas demonstraram risco de ocorrência de catarata abaixo de 19\% em homens que consomem altos níveis de luteína (BROWN et al., 1999).

\section{FUNÇÕES}

Os carotenoides, em consequência da sua estrutura conjugada de polienos, desempenham funções essenciais. 


\subsection{COLORAÇÃO}

Os carotenoides são responsáveis pela coloração de frutos e vegetais entre o amarelo e o vermelho (COOPER, ELDRIDGE e PETERS, 1999). A habilidade dos carotenoides de absorver luz na região visível e consequentemente de apresentar cor envolve a formação do estado singlete desse e se deve ao grande número de insaturações conjugadas presentes na molécula. Quanto maior o número de insaturações mais intensa é a cor do composto. Pelo menos sete duplas ligações conjugadas são necessárias para que o carotenoide seja colorido (KRINSKY, 1994; RODRIGUEZAMAYA, 1999).

\subsection{ATIVIDADE DE PRÓ-VITAMINA A}

A dieta proporciona vitamina A na forma de vitamina pré-formada a partir de alimentos de origem animal como, por exemplo, leite, pescado e carnes, ou como carotenoides que podem se transformar biologicamente em vitamina A, geralmente a partir de alimentos de origem vegetal. Estima-se que aproximadamente $60 \%$ da vitamina Ada dieta provêm dos carotenoides (RODRIGUEZAMAYA, KIMURA e AMAYA-FARFAN, 2008).

A transformação dos carotenoides pró-vitamínicos em vitamina A ocorre por clivagem central, sendo o carotenoide dividido ao meio, formando uma ou duas moléculas de retinal, que posteriormente é transformada em retinol (OLSON, 1999).

A vitamina A desempenha diversos papéis no organismo, destacando-se sua participação no processo da visão, no crescimento, no desenvolvimento e na manutenção do tecido epitelial, além das funções imunológica, de reprodução e do desenvolvimento embrionário (EL-BEITUNE et al., 2003; MELÉNDEZ-MARTÍNEZ, VICARIO e HEREDIA, 2004). $O$ excesso de vitamina $A$ pode ser nocivo, enquanto que os carotenoides pró-vitamínicos $A$, como o $\beta$-caroteno, não produz mal algum. O nível máximo de ingestão de vitamina $A$ recomendado é de $6 \mathrm{mg} /$ dia (PASSWATER, 1998).

A exigência mínima para o carotenoide apresentar atividade pró-vitamínica $A$ é ter um anel- $\beta$ não substituído, com uma cadeia poliênica de onze carbonos (RODRIGUEZ-AMAYA, KIMURA e AMAYA-FARFAN, 2008). Destaca-se com $100 \%$ de atividade pró-vitamínica $A$ o $\beta$-caroteno por ser capaz de originar, a partir da ruptura enzimática de sua estrutura, duas moléculas de vitamina A. Seguem a criptoxantina com 57\%, a-caroteno com 53\%, $\gamma$-caroteno entre $42-50 \%$ e o $\beta$-zeacaroteno, entre 20-40\% (MÍNGUEZ-MOSQUERA, HORNERO-MÉNDEZ e PÉREZ-GÁLVEZ, 2002).

\subsection{ATIVIDADE ANTIOXIDANTE}

As propriedades antioxidantes dos carotenoides fundamentam-se na estrutura desses compostos, principalmente no sistema de duplas ligações conjugadas, tornando possível a captação de radicais livres (TAPIERO, TOWNSEND e TEW, 2004; YOUNG e LOWE, 2001).

Os carotenoides participam do mecanismo de fotoproteção (Figura 2) sequestrando o oxigênio singlete. Essa reação envolve a transferência de energia entre o oxigênio singlete $\left({ }^{1} \mathrm{O}_{2}\right)$ e o carotenoide para gerar oxigênio triplete $\left({ }^{3} \mathrm{O}_{2}\right)$. O carotenoide energizado formado retorna ao estado fundamental dissipando essa energia mediante interações rotacionais com o sistema de solventes (CARIS-VEYRAT, 2007; SCHAFER et al., 2002). A energia liberada é baixa e, portanto, inofensiva ao meio celular. Esse mecanismo protege os portadores de porfirias cutâneas contra os efeitos nocivos do oxigênio singlete formado na pele a partir da exposição à luz solar. Tais efeitos protetores são indicados pela redução da concentração de $\beta$-caroteno na pele dos indivíduos afetados (MORTENSEN, SKIBSTED e TRUSCOTT, 2001; WOODALL, BRITON e JACKSON, 1997).

$$
\begin{array}{lll}
{ }^{1} \mathrm{O}_{2}+\text { Carotenoide } & \longrightarrow & { }^{3} \mathrm{O}_{2}+\text { Carotenoide energizado } \\
\text { Carotenoide energizado } & \longrightarrow & \text { Carotenoide }+ \text { calor }
\end{array}
$$


Os carotenoides podem reduzir as taxas de fotoxidação, a oxidação das lipoproteínas de baixa densidade e proteger o DNA contra o ataque dos radicais livres (BURRI et al., 1998; MOLLER, LOFT, 2004; PIMENTEL, FRANCKI e GOLLÜCKE, 2005). Também podem remover os radicais alquiperoxila (ROO'), sendo que in vitro isso ocorre por três vias (CARIS-VEYRAT, 2007; El-AGAMEY et al., 2004), como mostram as reações de:

a) Transferência de elétrons: Carotenoide $+\mathrm{ROO}^{*} \rightarrow$ Carotenoide $+\mathrm{ROO}$

b) Abstração de hidrogênio: Carotenoide $+\mathrm{ROO}^{*} \rightarrow$ Carotenoide $^{*}+\mathrm{ROOH}$

c) Adição: Carotenoide + ROO $\rightarrow$ ROO Carotenoide.

\subsection{ATIVIDADE PRÓ-OXIDANTE}

Uma molécula com efeito pró-oxidante pode ser definida como aquela que interage com espécies reativas de oxigênio (ROS) para formar compostos mais prejudiciais para a própria biomolécula (CARIS-VEYRAT, 2007; LOWE, VLISMAS e YOUNG, 2003). Burton e Ingold (1984) foram os primeiros a mencionar a atividade pró-oxidante dos carotenoides.

Existem três mecanismos principais pelos quais os carotenoides podem se transformar em pró-oxidantes (El-AGAMEY et al., 2004; LOWE, VLISMAS e YOUNG, 2003; YOUNG e LOWE, 2001):

a) Reações com ROS ou espécies reativas de nitrogênio (RNS) em que a molécula de carotenoide é oxidada e/ou clivada, gerando produtos pró-oxidantes:

b) Altas concentrações celulares de carotenoides podem aumentar a permeabilidade das membranas biológicas para toxinas e radicais;

c) Aumento da pressão de oxigênio ou altas concentrações de carotenoides podem levar a formação de um radical carotenoide peroxil, iniciando a lipoperoxidação.

Vários estudos demonstraram a perda da atividade antioxidante dos carotenoides como, por exemplo, o realizado por Galinato et al. (2007) sobre a autoxidação de carotenoides com metais. Tais autores propuseram mecanismo no qual o $\beta$-caroteno desapareceu após 10 minutos de reação com ferro, ou seja, ocorreu a autoxidação.

A oxidação de carotenoides por moléculas de oxigênio, ou seja, o processo de autoxidação constitui fenômeno complexo que está provavelmente relacionado com algum fator externo (metal, radical, etc.). A autoxidação dos carotenoides torna-se importante quando se estuda sua atividade antioxidante, pois se pode obter resultados não coerentes (CARIS-VEYRAT, 2007).

\section{IMPORTÂNCIA FISIOLÓGICA}

Os fatores que influenciam a atividade dos carotenoides em sistemas biológicos são: a estrutura (tamanho, natureza, posição e números de grupos substituintes), a forma física (agregados ou monômeros, configuração cis ou trans), a localização ou sítio de ação da molécula de carotenoide dentro da célula, o potencial de interação com outros carotenoides ou antioxidantes, a concentração e a pressão parcial de oxigênio (YOUNG e LOWE, 2001).

Há indícios de que os carotenoides em associação com outros componentes dos frutos apresentam efeito protetor contra algumas doenças crônicas. Por exemplo, o efeito sinergístico entre $\beta$-caroteno e as vitaminas $C$ e $E$ foi observado na proteção celular, provavelmente decorrente da capacidade do $\beta$-caroteno em destruir os radicais livres e reparar os radicais de tocoferol produzidos pela ação do $\alpha$-tocoferol (UENOJO, MARÓSTICA JUNIOR e PASTORE, 2007).

A partir da evidência científica, baseada em estudos epidemiológicos e em ensaios experimentais, e da elucidação dos mecanismos de atuação de fitoquímicos relacionados à maior proteção contra o câncer mostram que a alimentação rica em fontes hortifrutíferas de carotenoides representa possibilidade de proteção contra o desenvolvimento do câncer, e que a ingestão suplementar não reproduz essa proteção (COOPER, ELDRIDGE e PETERS, 1999).

\section{MÉTODOS DE DETERMINAÇÃO}

A necessidade de dados confiáveis sobre carotenoides em alimentos é largamente reconhecida em diversos campos de estudo. Os fatores que tornam essa análise difícil incluem o grande número de carotenoides naturalmente existentes, a variação quantitativa e qualitativa desses 
nos alimentos, a pouca quantidade de carotenoides pró-vitamínicos $\mathrm{A}$, a sua variada biopotência e o fato dos carotenoides serem moléculas extremamente insaturadas, propiciando processos de isomerização, oxidação e degradação durante a análise (RODRIGUEZ-AMAYA, 2001; RODRIGUEZAMAYA, KIMURA e AMAYA-FARFAN, 2008).

Em geral, a análise de carotenoides consiste na amostragem e preparação da amostra, extração (mistura de solventes, fluído supercrítico, etc.), partição em solvente compatível para a cromatografia, saponificação e lavagem, concentração ou evaporação do solvente e identificação e quantificação. Todas essas etapas estão sujeitas a erros, cujos mais comuns são a amostragem não representativa, extração incompleta, perdas físicas durante as etapas e perda de carotenoides na lavagem (RODRIGUEZ-AMAYA, 2001).

A análise de carotenoides nos alimentos pode ser realizada de forma global, determinandose apenas de forma quantitativa os compostos presentes na matriz alimentar ou da forma que permite identificar os carotenoides presentes na amostra. Existem várias técnicas para essas determinações, embora todas estejam sujeitas a várias fontes de erros (RODRIGUEZ-AMAYA, KIMURA e AMAYA-FARFAN, 2008).

\subsection{ESPECTROFOTOMETRIA}

A forma global de identificação dos carotenoides baseia-se na determinação espectrofotométrica da amostra, efetuando-se leitura da absorbância do extrato obtido na etapa de extração no comprimento de onda de máxima absorção do composto, calculando-se em seguida a sua concentração. A grande vantagem desse método sobre os gravimétricos e volumétricos é que se pode determinar com maior exatidão e de maneira simples traços desses compostos presentes em alimentos (ROZZI et al., 2002).

\subsection{ESPECTROMETRIA DE MASSA}

Ferramenta analítica poderosa, a espectrometria da massa é capaz de identificar compostos desconhecidos, quantificar materiais conhecidos e elucidar as propriedades químicas e estruturais das moléculas. A detecção pode ser obtida para quantidades pequenas, como $10^{-15} \mathrm{~g}$, ou para composto de massa de 1.000 Daltons em misturas quimicamente complexas. Para se obter maior potencialidade, essa técnica deve ser acoplada com técnicas cromatográficas (DASS, 2007).

A essência da técnica envolve a geração de íons que são depois detectados, permitindo a obtenção de informação sobre a massa molecular e as características estruturais dos carotenoides (HARRIS, 2008).

\subsection{CROMATOGRAFIA A LÍQUIDO DE ALTA EFICIÊNCIA}

A cromatografia a líquido de alta eficiência constitui processo de análise de separação física, cuja aplicação permite a determinação qualitativa e quantitativa das amostras. Esse método vem sendo amplamente empregado graças à alta precisão, ao tempo relativamente rápido de execução, à sua boa sensibilidade e à possibilidade de separar espécies não voláteis e termicamente instáveis. A separação dos constituintes de determinada mistura é realizada através de sua distribuição em duas fases: estacionária e móvel. Essa técnica é capaz de promover a separação dos carotenoides, porém não consegue separar suas respectivas formas isoméricas (OLIVEIRA, 2009). Pode-se usar colunas de fase direta ou reversa, sendo que a diferença está na polaridade das fases estacionária e móvel. O sistema de fase reversa apresenta vantagens, como o uso de fases móveis menos tóxicas e de menor custo, o rápido equilíbrio da coluna após a mudança da fase móvel, a facilidade de emprego de eluição por gradiente, a maior rapidez em análises e a boa reprodutibilidade dos tempos de retenção (TONHI et al., 2002).

\subsection{CROMATOGRAFIAA GÁS}

A cromatografia a gás serve para separação e análise de misturas de substâncias voláteis, muito utilizada para determinar o perfil de carotenoides. Nesse método, a amostra é vaporizada e introduzida em fluxo de gás adequado denominado de fase móvel ou gás de arraste. Esse fluxo de gás com a amostra vaporizada passa por coluna contendo a fase estacionária em que ocorre a separação da mistura. As substâncias separadas saem da coluna dissolvidas no gás de arraste e passam por dispositivo que gera sinal elétrico proporcional à quantidade de material eluído. $\mathrm{O}$ 
registro desse sinal em função do tempo é o cromatograma, sendo que as substâncias aparecem nele como picos com área proporcional à sua massa, o que possibilita a análise quantitativa (SCOTT e PERRY, 1997).

\subsection{COLORIMETRIA}

Técnica simples e rápida, a colorimetria tem sido empregada em larga escala para a seleção de genótipos com maiores teores de carotenoides, evitando o desperdício e os problemas de descarte dos solventes. No entanto, apresenta menor precisão se comparada com outros métodos. Nessa técnica, a concentração de componente específico da amostra é determinada pela comparação da intensidade de cor da amostra com o padrão. A amostra colorida tem características especiais de absorção de luz que estão relacionadas com o conteúdo de carotenoides (CARVALHO et al., 2005).

\section{CONCLUSÃO}

Os carotenoides estão presentes em muitos vegetais e podem ser analisados por diversos métodos. Acredita-se que a melhor técnica analítica para avaliá-los seja a cromatografia a líquido de alta eficiência acoplada à espectrometria de massa, pois além de quantificar e qualificar os carotenoides, detecta aqueles presentes em menores quantidades.

A alimentação humana é pobre em frutas e verduras ricas em carotenoides, que fornecem vários benefícios ao organismo humano por agirem como antioxidantes e pró-vitamínicos $A$. Recomenda-se o consumo de abóboras por apresentarem quantidades relevantes de carotenoides importantes, como $\alpha$-caroteno, $\beta$-caroteno e luteína.

\section{ABSTRACT}

\section{CAROTENOIDS IN PUMPKINS}

The present literature review highlights the importance of carotenoids as bioactive compound, and its potential application in functional foods. It were detailed the origin, chemical characteristics and sources of the carotenoids. It were also studied the most used methods to quantify and qualify the carotenoids, including spectrophotometry, mass spectrometry, high-performance liquid chromatography, gas chromatography and colorimetry. The pumpkin intake is recommended because they represent important source of carotenoids such as $\alpha$-carotene, $\beta$-carotene and lutein.

KEY-WORDS: CAROTENOIDS; PUMPKINS; ANTIOXIDANT; PRO-VITAMIN A.

\section{REFERÊNCIAS}

1 AMBRÓSIO, C.L.B.; CAMPOS, F.A.C.S.; FARO, Z.P. Carotenóides como alternativa contra a hipovitaminose A. Revista Nutrição, Campinas, v. 19, n. 2, p. 233-243, mar./abr. 2006.

2 ARIMA, H.K.; RODRÍGUEZ-AMAYA D.B. Carotenoid composition and vitamin A value of a squash and a pumpkin from northeastern Brazil. Archivos Latinoamericanos de Nutrición, Caracas, v. 40, n. 2, p. 284-292, jun. 1990.

3 ARUNA, G.; MAMATHA, B.S.; BASKARAN, V. Lutein content of selected Indian vegetables and vegetables oils determined by HPLC. Journal of Food Composition and Analysis, Rome, v. 22, n. 1, p. 632-636, Jul. 2009.

4 BERNHARDT, S.; SCHLICH, E. Impact of different cooking methods on food quality: retention of lipophilic vitamins in fresh and frozen vegetables. Journal of Food Engineering, Amsterdam, v. 77, n. 1, p. 327-333, Nov. 2006.

5 BURRI, B.J. Beta-carotene and human health: a review of current research. Nutrition Research, Chair, v. 17, n. 3, p. 547-580, Mar. 1997.

6 BURRI, B.J. et al. Estimating the concentration of beta-carotene required for maximal protection of low-density lipoproteins in women. American Journal of Clinical Nutrition, Bethesda, v. 67, n. 1, p. 837-845, Nov. 1998.

7 BURTON, G. W.; INGOLD, K. U. Beta-carotene: an unusual type of lipid antioxidant. Science, Lancaster, v. 224, n. 1, p. 569-573, May 1984. 
8 BROWN, L. et al. A prospective study of carotenoid intake and risk of cataract extraction in U. S. men. American Journal of Clinical Nutrition, Bethesda, v. 70, n. 1, p. 517-524, Oct. 1999.

9 CARIS-VEYRAT, C. Antioxidant and prooxidant actions and stabilities of carotenoids in vitro and in vivo and carotenoid oxidation products. In: SOCACIU, C. Food colorants: chemical and function properties. Washington: CRC Press, 2007. p. 177-195.

10 CARVALHO, W. et al. Estimativa indireta de teores de licopeno em frutos de genótipos de tomateiro via análise colorimétrica. Horticultura Brasileira, Brasília, v. 23, n. 3, p. 819-825, set. 2005.

11 COOPER, D.A.; ELDRIDGE, A.L.; PETERS, J.C. Dietary carotenoids and lung cancer: a review of recent research. Nutrition Reviews, New York, v. 57, n. 5, p. 133-145, May 1999.

12 DASS, C. Fundamentals of contemporary mass spectrometry. San Francisco: Wiley-Interscience, 2007.608 p.

13 DUTTA D.; CHAUDHURI, U.R.; CHAKRABORTY, R. Structure, health benefits, antioxidant property and processing and storage of carotenoids. African Journal of Biotechnology, Calcutá, v. 4, n. 13, p. 1510-1520, Dec. 2005.

14 El-AGAMEY, A. et al. Carotenoid radical chemistry and antioxidant/pro-oxidant properties. Archives Biochemistry and Biophysics, New York, v. 430, n. 1, p. 37-48, Oct. 2004.

15 EL-BEITUNE, P. et al. Deficiência da vitamina A e associações clínicas: revisão. Archivos Latinoamericanos de Nutrición, Caracas, v. 53, n. 4, p. 355-363, Nov. 2003.

16 FILGUEIRA, F.A.R. Novo manual de olericultura: agrotecnologia moderna na produção e comercialização de hortaliças. Viçosa: Universidade Federal de Viçosa, 2000. 402 p.

17 GALINATO, M.G.I. et al. Cation radicals of xanthophylls. Photosynthesis Research, Netherlands, v. 94, n. 1, p. 67-78, Jul. 2007.

18 HARRIS, D. C. Análise química quantitativa. 7. ed. Rio de Janeiro: LTC, 2008. 886 p.

19 HUGHES, C. Guia de aditivos. Zaragoza: Acribia, 1994. 200 p.

20 KANDALAKUNTA, B.; RAJENDRAN, A.; THINGNGANING, L. Carotene content of some common (cereals, pulses, vegetables, spices and condiments) and unconventional sources of plant origin. Food Chemistry, London, v. 106, n. 1, p. 85-89, May 2008.

21 KRINSKY, N.I. The biological properties of carotenoids. International Union of Pure and Applied Chemistry, Genebra, v. 66, n. 5, p. 1003-1010, Jan. 1994

22 LANDRUM, J. T.; BONE, R. A. Lutein, zeaxanthin and the macular pigment. Archives Biochemistry and Biophysics, New York, v. 28, n. 1, p. 28-40, Jan. 2001.

23 LOWE, G. M.; VLISMAS, K.; YOUNG, A. J. Carotenoids as prooxidants? Molecular Aspects of Medicine, Oxford, v. 24, n. 6, p. 363-369, Dec. 2003

24 LUENGO, R.F.A.; CALBO, A.G. Armazenamento de hortaliças. Brasília: Empresa Brasileira de Pesquisa Agropecuária - Embrapa Hortaliças, 2001. 242 p.

25 MATTEA, F.; MARTÍN, A.; COCERO, M. J. Carotenoid processing with supercritical fluids. Journal of Food Engineering, Amsterdam, v. 93, n. 1, p. 255-265, Aug. 2009.

26 MELÉNDEZ-MARTÍNEZ, A.J.; VICARIO, I.M.; HEREDIA, F.J. Importancia nutricional de los pigmentos carotenóides. Archivos Latinoamericanos de Nutrición, Caracas, v. 54, n. 2, p. 149-155, Jun. 2004.

27 MEYERS, P.S. Developments in world aquaculture, feed formulation and role of carotenoids. International Union of Pure and Applied Chemistry, Genebra, v. 66, n. 5, p. 1069-1076, Jan. 1994.

28 MÍNGUEZ-MOSQUERA, M.I.; HORNERO-MÉNDEZ, D.; PÉREZ-GÁLVEZ, A. Carotenoids and provitamin A in functional foods. In: HURST, W.J. Methods of analysis for functional foods and nutraceuticals. Washington: CRC Press, 2002. p. 101-158.

29 MOLLER, P.; LOFT, S. Interventions with antioxidants and nutrients in relation to oxidative DNA damage and repair. Mutation Research: fundamental and molecular mechanisms of mutagenesis, v. 551, n. 1-2, p. 79-89, Jul. 2004.

30 MORTENSEN, A.; SKIBSTED, L.H.; TRUSCOTT, T.G. The interaction of dietary carotenoids with radical species. Archives Biochemistry and Biophysics, New York, v. 385, n. 1, p. 13-19, Jan. 2001.

31 MURKOVIC, M.; MÜLLEDER, U.; NEUNTEUFL, H. Carotenoid content in different varieties of pumpkins. Journal of Food Composition and Analysis, Rome, v. 15, n. 1, p. 633-638, Dec. 2002 
32 NISHINO, H. et al. Carotenoids in cancer chemoprevention. Cancer and Metastasis Reviews, v. 21, n. 4, p. 257-264, Jun. 2002.

33 OLIVEIRA, C.G. Purificação parcial de beta-caroteno usando suco de caju como substrato. In: CONGRESSO BRASILEIRO DE ENGENHARIA QUÍMICA EM INICIAÇÃO CIENTÍFICA (COBEQ IC), 8., 2009, Uberlândia. Resumos... Uberlândia: Faculdade de Engenharia Química, 2009.

34 OLIVER, J.; PALOU, A. Chromatographic determination of carotenoids in foods. Journal of Chromatography A, Amsterdam, v. 881, n. 1, p. 543-555, Jun. 2000.

35 OLSON, J.A. Bioavailability of carotenoids. Archivos Latinoamericanos de Nutrición, Caracas, v. 49, n. 1, p. 21-25, Set. 1999.

36 ÖTLES, S.; ÇAGINDI, Ö. Carotenoids as natural colorants. In: SOCACIU, C. Food colorants: chemical and function properties. Washington: CRC Press, 2007. p. 51-70.

37 PASSWATER, R.A. All about antioxidants. New York: Avery Publishing Group, 1998. 96 p.

38 PIMENTEL, C.V.M.B.; FRANCKI, V.M.; GOLLÜCKE, A.P.B. Alimentos funcionais. São Paulo: Varela, 2005.95 p.

39 ROCK, C. L. Carotenoids: biology and treatment. Clinical Pharmacology and Therapeutics, Saint Louis, v. 6, n. 3, p. 185-197, Sept. 1997

40 RODRIGUEZ-AMAYA, D. B. Latin american food sources of carotenoids. Archivos Latinoamericanos de Nutrición, Caracas, v. 49, n. 3, p. 74S-84S, Set. 1999.

41 RODRIGUEZ-AMAYA, D.B. A guide to carotenoid analysis in foods. Washington: ILSI Press, 2001. 64 p.

42 RODRIGUEZ-AMAYA, D. B.; KIMURA, M.; AMAYA-FARFAN, J. Fontes de carotenóides: tabela brasileira de composição de carotenóides em alimentos. Brasília: Ministério de Meio Ambiente/Secretaria de Biodiversidade e Florestas, 2008. 99 p.

43 ROZZI, N.L. et al. Supercritical fluid extraction of lycopene from tomato processing byproducts. Journal of Agricultural and Food Chemistry, Easton, v. 50, n. 1, p. 2638-2643, Mar. 2002.

44 SANDERS, T.A.B. Dietary fat-weighing up the pros and cons. Nutrition \& Food Science, London, v. 94, n. 5, p. 9-13, Nov. 1994

45 SCHAFER, F.Q. et al. Comparing $\beta$-carotene, vitamin E and nitric oxide as membrane antioxidants. Journal of Biological Chemistry, Bethesda, v. 277, n. 1, p. 671-681, Nov. 2002.

46 SCOTT, R.P.W.; PERRY, J.A. Introduction to analytical gas chromatography. $2^{\text {nd }}$ ed. New York: Marcel Deckker, 1997. $416 \mathrm{p}$.

47 SEO, J.S. et al. Extraction and chromatography of carotenoids from pumpkin. Journal of Chromatography A, Amsterdam, v.1073, n. 1, p. 371-375, May 2005.

$48 \mathrm{SHI}$, J. et al. Effects of supercritical $\mathrm{CO}_{2}$ fluid parameters on chemical composition and yeld of carotenoids extracted from pumpkin. Food Science and Technology, Zurique, v. 49, n. 1, p. 39-44, Jan. 2010.

49 SHILLS, M.E. et al. Tratado de nutrição moderna na saúde e na doença. 9. ed. São Paulo: Manole, 2003.1026 p.

50 SILVA, A.S. Luteína, alimento para tu vista. Food Ingredients, São Paulo, v. 6, n. 1, p. 80-81, Jul. 2004.

51 SIQUEIRA, E. M. A. et al. $\beta$-caroteno da folha de mandioca (Manihot Esculenta Crantz) melhora o estado nutricional de vitamina A em ratos. Comparative Biochemistry and Physiology, New York, v. 146, n. 2, p. 235-240, Nov. 2007.

52 TAPIERO, H.; TOWNSEND, D.M.; TEW, K.D. The role of carotenoids in the prevention of human pathologies. Biomedicine \& Pharmacotherapy, Paris, v. 58, n. 2, p. 100-110, Mar. 2004.

53 THANE, C.; REDDY, S. Processing of fruit and vegetables: effects on carotenoids. Nutrition \& Food Science, London, v. 97, n. 2, p. 58-65, Apr. 1997.

54 TONHI, E. et al. Fases estacionárias para cromatografia líquida de alta eficiência em fase reversa (CLAE-FR) baseadas em superfícies de óxidos inorgânicos funcionalizados. Química Nova, São Paulo, v. 25, n. 4, p. 616-623, jul. 2002.

55 UENOJO, M.; MARÓSTICA JUNIOR, M.R.N.; PASTORE, G.M. Carotenóides: propriedades, aplicações e biotransformação para formação de compostos de aroma. Química Nova, São Paulo, v. 30, n. 3, p. 616-622, fev. 2007.

56 WOODALL, A.A.; BRITON, G.; JACKSON, M.J. Carotenoids and protection of phospholipids in solution or in liposomes against oxidation by peroxyl radicals: relationship between carotenoid structure and protective ability. Biochimica et Biophysica Acta (BBA)-General Subjects, Amsterdam, v. 1336, n. 3, p. 575-586, Oct. 1997. 
57 YOUNG, A.J.; LOWE, G.M. Antioxidant and prooxidant properties of carotenoids. Archives Biochemistry and Biophysics, New York, v. 385, n.1, p. 20-27, Jan. 2001.

58 ZITTER, T.; HOPKINS, D. L.; THOMAS, C. E. Compendium of cucurbit diseases. Minnesota: Academic Press, 1996. 148 p. 